Portland State University

PDXScholar

Electrical and Computer Engineering Faculty

Publications and Presentations

8-1-2009

\title{
Gain Saturation in Gain-Guided Slab Waveguides with Large-Index Antiguiding
}

Tsing-Hua Her

University of North Carolina at Charlotte

Xianyu Ao

University of North Carolina at Charlotte

Lee W. Casperson

Portland State University

Follow this and additional works at: https://pdxscholar.library.pdx.edu/ece_fac

Part of the Electrical and Computer Engineering Commons

Let us know how access to this document benefits you.

Citation Details

Tsing-Hua Her, Xianyu Ao, and Lee W. Casperson, "Gain saturation in gain-guided slab waveguides with large-index antiguiding," Opt. Lett. 34, 2411-2413 (2009).

This Article is brought to you for free and open access. It has been accepted for inclusion in Electrical and Computer Engineering Faculty Publications and Presentations by an authorized administrator of PDXScholar. Please contact us if we can make this document more accessible: pdxscholar@pdx.edu. 


\title{
Gain saturation in gain-guided slab waveguides with large-index antiguiding
}

\author{
Tsing-Hua Her, ${ }^{1, *}$ Xianyu Ao, ${ }^{1}$ and Lee W. Casperson ${ }^{2}$ \\ ${ }^{1}$ Department of Physics and Optical Science, and Center for Optoelectronics and Optical Communications, \\ The University of North Carolina at Charlotte, 9201 University City Boulevard, \\ Charlotte, North Carolina 28223, USA \\ ${ }^{2}$ Department of Electrical and Computer Engineering, and Center for Optoelectronics and Optical Communications, \\ The University of North Carolina at Charlotte, 9201 University City Boulevard, \\ Charlotte, North Carolina 28223, USA \\ *Corresponding author: ther@uncc.edu
}

Received April 12, 2009; accepted June 17, 2009;

posted July 20, 2009 (Doc. ID 109606); published August 4, 2009

\begin{abstract}
We investigate numerically and analytically the effects of gain saturation on the propagation of the fundamental mode in a gain-guided index-antiguided slab waveguide. The propagating mode adapts to gain saturation by becoming less confined, while at the same time its peak intensity increases more slowly. At steady state, both the mode shape and the power remain constant. (C) 2009 Optical Society of America

OCIS codes: $140.3280,230.7400$.
\end{abstract}

Waveguides with large mode area are highly desired for high-power laser oscillators and amplifiers to mitigate optical nonlinearity and damage. Research on this subject remains active, and many new approaches have been pursued [1-4]. Siegman proposed the use of gain guiding (GG) in index-antiguided (IAG) waveguides to achieve large-area singletransverse-mode propagation [5,6], and single-mode laser oscillation in GG-IAG fibers with core diameters up to $400 \mu \mathrm{m}$ has been experimentally demonstrated $[7,8]$. In GG-IAG waveguides, all modes are intrinsically leaky with higher loss for higher-order modes. To achieve single-transverse-mode operation, an optical gain that is higher than the threshold gain of the fundamental mode but below those of higherorder modes is provided such that only the fundamental mode is confined and amplified [5]. Waveguides (fibers) with strong index antiguiding are particularly attractive, because they substantially reduce the gain threshold for gain guiding [6], thus making GG more practical in terms of fabrication and pumping requirements.

Extensive analysis has been performed for GG-IAG waveguides with constant and uniform gain in the core [6]. In practical high-power laser amplifiers and oscillators, however, gain is subject to saturation effects in both longitudinal and transverse directions, whether the gain medium is homogeneously or inhomogeneously broadened [9]. Since GG relies on gain to sustain a confined mode [10], it is important to understand the effect of gain saturation on the modal stability in GG-IAG waveguides. In this Letter, we investigate the propagation characteristics of the fundamental mode in an IAG slab waveguide with gain saturation of homogeneous line broadening in the core.

Consider a GG-IAG slab waveguide with a core width $2 a$ and a refractive index $n_{1}$ that is slightly smaller than that of the cladding $n_{2}$. The waveguide extends infinitely along the $z$ direction and has a uniform small-signal (unsaturated) power gain coeffi- cient $g_{s s}$ only inside the core. The complex refractive index profile $\tilde{n}(x, z)$ at line center is given by

$$
\tilde{n}(x, z)=\left\{\begin{array}{ll}
n_{1}-j \frac{n_{s s}}{1+s I(x, z)} & (|x| \leqslant a) \\
n_{2} & (|x|>a)
\end{array},\right.
$$

where $I(x, z)$ is the intensity, $s$ is the saturation parameter defined as the inverse of the saturation intensity, $n_{s s}$ is related to $g_{s s}$ by $g_{s s}=2 k_{o} n_{s s}$, and $k_{o}$ is the free-space wavenumber. For weak index contrast, we may consider only the scalar electric field $\widetilde{E}(x, z, t)$. When the signals are small such that gain saturation is negligible, the waveguide is longitudinally uniform, and its eigenmodes $\widetilde{\psi}(x)$, defined by $\widetilde{E}=\widetilde{\psi}(x) \exp [j(\tilde{\beta} z-\omega t)]$ where $\widetilde{\beta}=\beta_{r}+j \beta_{i}$ is the complex propagation constant and $\omega$ is the frequency, can be solved analytically [5]. The fundamental mode bears the following form:

$$
\tilde{\psi}(x)=\left\{\begin{array}{ll}
\cos (\tilde{u} x / a) & (|x| \leqslant a) \\
\cos (\widetilde{u}) e^{-\widetilde{w}(|x| / a-1)} & (|x| \geqslant a)
\end{array},\right.
$$

where $\widetilde{u}=a \sqrt{k_{0}^{2}\left(n_{1}-j n_{s s}\right)^{2}-\widetilde{\beta}^{2}}$ and $\widetilde{w}=a \sqrt{\widetilde{\beta}^{2}-k_{0}^{2} n_{2}^{2}}$ are the modal parameters in the core and cladding, respectively. As the signal intensity increases, gain decreases and the complex index profile $\widetilde{n}(x, z)$ becomes nonuniform in both transverse and longitudinal directions. We solve for the envelope function $\widetilde{\phi}(x, z)$ of the electric field $\widetilde{E}=\widetilde{\phi}(x, z) \exp [j(\bar{k} z-\omega t)]$ where $\bar{k}$ is the reference wavenumber, using a finite-difference beam-propagation method (BPM) with perfectly matched layers as boundary conditions $[11,12]$. The launch field is the unsaturated fundamental mode $\widetilde{\psi}(x)$ with unit amplitude, and $\bar{k}$ is taken to be $\beta_{r}$. During the propagation, the gain profile in Eq. (1) is updated using the intensity of the previous $z$ step. 
Below we consider a numerical example with $n_{1}$ $=1.469, n_{2}=1.470,2 a=100 \mu \mathrm{m}, \lambda=1.53 \mu \mathrm{m}$, and $s I(x, z)=0.0025|\widetilde{\phi}(x, z)|^{2}$. Such a waveguide has a dimensionless index parameter $-\Delta N=120$, where $\Delta N$ is defined in [6], which corresponds to reasonably strong index-antiguiding. The effective index of the fundamental mode of this (passive) waveguide is $1.46898+j 3.51 \times 10^{-6}$, and the imaginary part corresponds to a loss coefficient $0.288 \mathrm{~cm}^{-1}$. In the BPM simulation, a step size of $0.1 \mu \mathrm{m}$ is used for both $x$ and $z$ directions.

Figure 1(a) shows the evolution of the field over a distance of $500 \mathrm{~mm}$ for $g_{s s}=1.0 \mathrm{~cm}^{-1}$ (i.e., $n_{s s}$ $=1.2175 \times 10^{-5}$ ). In the first $100 \mathrm{~mm}$, the peak amplitude increases exponentially with distance and then saturates to a constant value at around $200 \mathrm{~mm}$. Beyond saturation, the field amplitude in the cladding is nearly constant in the $x$ direction, mimicking a cutoff-like mode. To examine the field evolution closely, the normalized field amplitudes at propagation distances of 25,100 , and $200 \mathrm{~mm}$ are shown in Fig. 1(b), with their corresponding gain profiles shown in Fig. 1(c). The latter clearly indicates a dip in the center of the gain profile due to gain saturation, and this dip increases with distance as a result of gain saturation. We calculate the net gain coefficient from successive traces in Fig. 1(a), defined as $g_{m}=P_{c}^{-1} \Delta P_{c} / \Delta z$, where $P_{c}$ is the power inside the core and $\Delta z$ is the step size, as a function of propagation distance. Shown as a black solid line in Fig. 2, the net-gain coefficient is nearly constant before $50 \mathrm{~mm}$, beyond which gain saturation depletes the gain exponentially. This can be understood by considering the net-gain coefficient of a plane wave in the presence of gain saturation and loss,
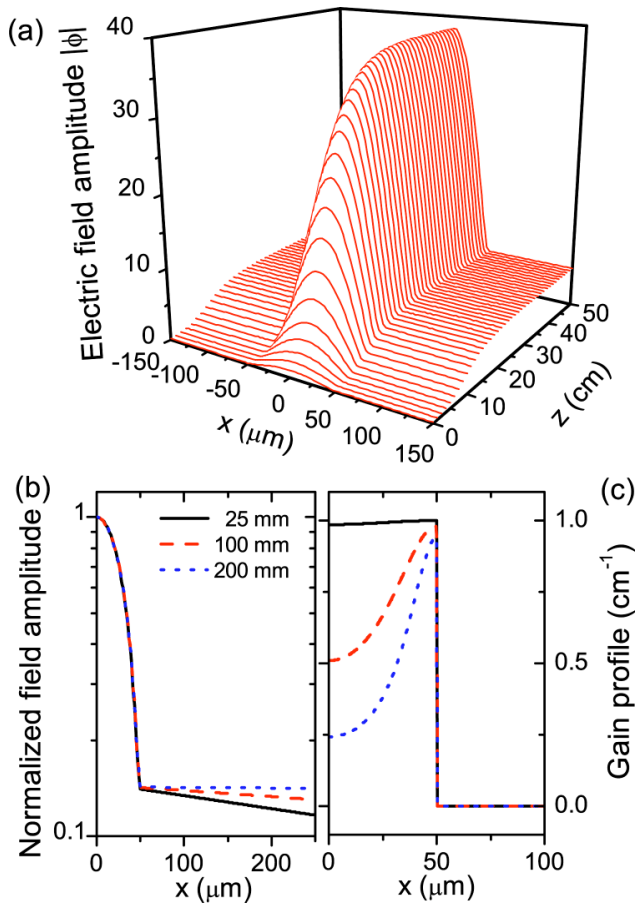

Fig. 1. (Color online) (a) Evolution of the electric field amplitude in a GG-IAG waveguide as described in the text. (b) Normalized field amplitudes and (c) the corresponding gain profiles at several distances along the waveguide.

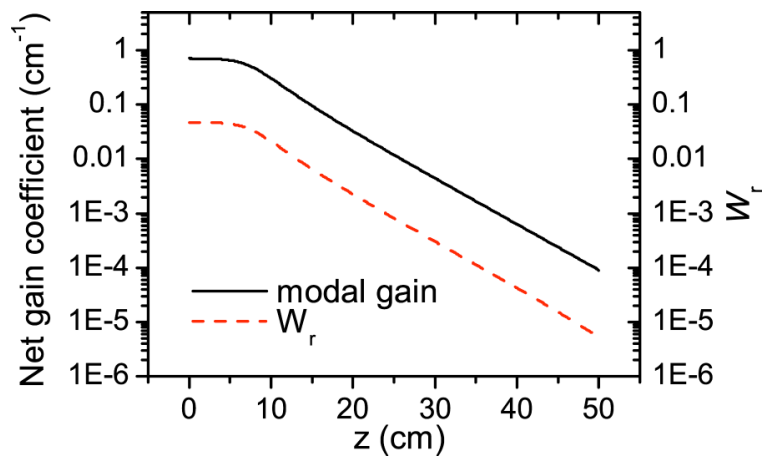

Fig. 2. (Color online) Net-gain coefficient and modal parameter $w_{r}$ in the cladding as functions of propagation distance.

$$
g(z)=\frac{1}{I(z)} \frac{\mathrm{d} I(z)}{\mathrm{d} z}=\frac{g_{s s}}{1+s I(z)}-\alpha,
$$

where $\alpha$ is the distributed loss coefficient. For small signals, $g \approx g_{s s}-\alpha$ is a constant, while at large intensity the net-gain coefficient is shown to decay exponentially according to

$$
\frac{1}{g(z)} \frac{\mathrm{d} g}{\mathrm{~d} z} \approx-\frac{g_{s s} \alpha}{g_{s s}-\alpha} .
$$

At different stages of gain saturation, Fig. 1(b) indicates that the field profiles still bear the same form as Eq. (2). In particular, the (normalized) field profiles inside the core have a nearly identical shape as the unsaturated mode, while those in the cladding become less confined with increasing propagation distance. The decay length of the cladding field is proportional to the real part of the cladding mode parameter $w_{r}(=\operatorname{Re}[\widetilde{w}])$ and can be calculated from Fig. 1 (a) as a function of propagation distance (dotted line in Fig. 2). As indicated, $w_{r}$ is linearly proportional to the net gain coefficient $g_{m}$ over the entire distance up to $500 \mathrm{~mm}$. As gain saturation depletes the net gain, the field in the cladding becomes less confined, leading asymptotically toward a cutoff-like mode as the net gain approaches zero. This linear relationship can be explained by expanding the phase-matching condition in the cladding with large $-\Delta N$ to be

$$
\frac{w_{r}}{a} \approx \frac{a \beta_{r} \beta_{i}}{\sqrt{-\Delta N}} \approx \frac{k_{o} a n_{1}}{2 \sqrt{-\Delta N}} g_{m} .
$$

Expression (5) indicates that the decay of field amplitude in the cladding is intrinsically proportional to its gain coefficient. This is different from conventional index-guided waveguides where the field decay in the cladding is determined solely by the index contrast.

The above observation indicates that the gainguided fundamental mode in a strongly IAG waveguide is robust against gain saturation. This is consistent with the previous report that the field distribution in a GG-IAG fiber is largely determined by the index antiguiding if the dimensionless index parameter $-\Delta N>100$ [6]. As gain saturation becomes significant, the field in the cladding adapts to lower 
gain by becoming less confined. When the gain is completely depleted, the cladding field stays cutofflike without turning into a mode with diverging amplitude in the cladding.

One important parameter in a laser amplifier is its maximum output power. Expression (3) describes the intensity evolution of a plane wave in a single-pass amplifier. For a nonuniform field, Expression (3) can be modified to calculate the total power along the propagation direction, provided that the normalized transverse profile remains constant [13]. Although the gain-guided mode in an IAG waveguide evolves continuously toward a cutoff-like mode, its shape in the core remains nearly constant. We can calculate the total power contained in the core, $P_{c}(z)$ $=\int_{-a}^{a} I(x, z) \mathrm{d} x$, as follows:

$$
\frac{1}{P_{c}(z)} \frac{\mathrm{d} P_{c}(z)}{\mathrm{d} z}=g_{s s} \int_{-a}^{a} \frac{f_{c}(x)}{1+s P_{c}(z) f_{c}(x)} \mathrm{d} x-\alpha,
$$

where $f_{c}(x)$ is the normalized intensity profile inside the core, defined by $\int_{-a}^{a} f_{c}(x) \mathrm{d} x=1$. The first term in the right-hand side of Eq. (6) can be regarded as the effective gain of the mode in the waveguide. In the current example, $\tilde{\psi}(x)=\cos (\tilde{u} x / a)$ in the core where $\tilde{u}=1.55755-j 0.139$, and Eq. (6) can be solved analytically if we substitute $f_{c}(x) \approx|\tilde{\psi}(x)|^{2} / a \approx \cos ^{2}(\pi x / 2 a) / a$ (the details will be reported elsewhere). The result is shown as the solid curve in Fig. 3, where $\alpha$ is replaced by the loss coefficient of the fundamental mode in the passive waveguide, which has been shown to be approximately equal to the threshold gain $g_{\text {th }}$ for a strongly IAG waveguide [6]. As a comparison, the evolution of power in the core as a function of propagation distance obtained from BPM is also shown (circles). The two methods are consistent (to within about $1.5 \%$ ), which suggests that the pas-

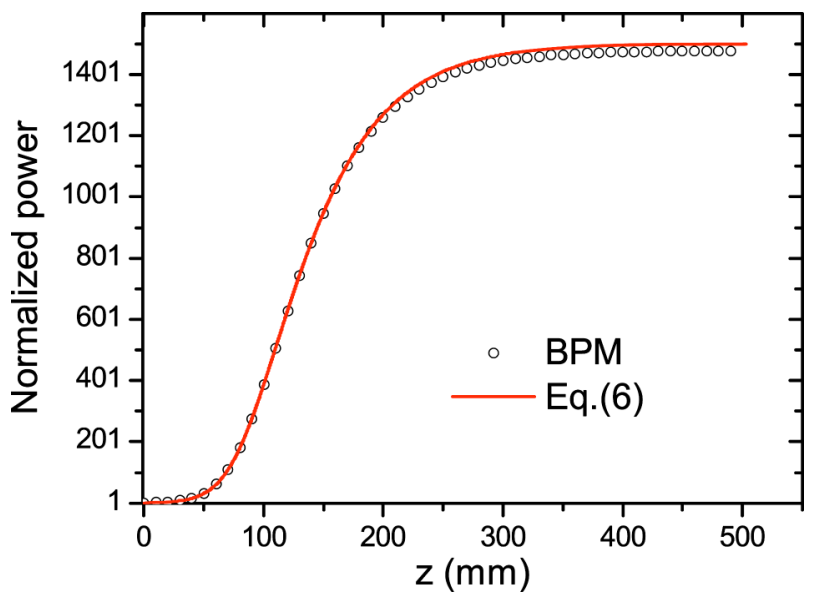

Fig. 3. (Color online) Normalized power as a function of propagation distance obtained from BPM simulation and from Eq. (6). sive modal loss in a strongly antiguided waveguide, which is due to diffraction of the wave away from the core, can be fairly accurately treated as the distributed loss of the waveguide in the presence of gain. With this approximation, other aspects of the fields and gain profiles can also be calculated analytically, and as an important brief example the maximum limiting power in the core is found to be

$$
P_{c, \max }=\frac{2 a}{s}\left[\left(\frac{g_{s s}}{g_{\mathrm{th}}}-\frac{1}{4}\right)-\sqrt{\frac{g_{s s}}{2 g_{\mathrm{th}}}+\frac{1}{16}}\right] .
$$

In conclusion, we have investigated numerically and analytically the effects of gain saturation on GG in a strongly IAG waveguide. We show that gain saturation has little effect on the field distribution in the core, whereas this saturation causes exponential decay of both the modal gain and the mode confinement. Steady state is reached when the effective gain balances the diffraction loss, where the mode becomes cutoff-like and maximum power is reached. Because the field distribution in the core is almost unchanging, the power and gain profiles can also be modeled analytically.

We would like to thank Professor Hung-Chun Chang of National Taiwan University for useful discussions. T. Her acknowledges financial support from a DARPA Young Faculty Award (HR0011-08-1-0065) and from National Science Foundation (NSF) under grant 0925992.

\section{References}

1. L. Dong, X. Peng, and J. Li, J. Opt. Soc. Am. B 24, 1689 (2007).

2. J. Limpert, N. Deguil-Robin, I. Manek-Honninger, F. Salin, F. Roser, A. Liem, T. Schreiber, S. Nolte, H. Zellmer, A. Tunnermann, J. Broeng, A. Petersson, and C. Jakobsen, Opt. Express 13, 1055 (2005).

3. S. Fevrier, D. D. Gaponov, P. Roy, M. E. Likhachev, S. L. Semjonov, M. M. Bubnov, E. M. Dianov, M. Y. Yashkov, V. E. Khopin, M. Y. Salganskii, and A. N. Guryanov, Opt. Express 14, 562 (2006).

4. A. Shirakawa, H. Maruyama, K. Ueda, C. B. Olausson, J. K. Lyngso, and J. Broeng, Opt. Express 17, 447 (2009).

5. A. E. Siegman, J. Opt. Soc. Am. B 20, 1617 (2003).

6. A. E. Siegman, J. Opt. Soc. Am. B 24, 1677 (2007).

7. A. E. Siegman, Y. Chen, V. Sudesh, M. C. Richardson, M. Bass, P. Foy, W. Hawkins, and J. Ballato, Appl. Phys. Lett. 89, 251101 (2006).

8. Y. Chen, T. McComb, V. Sudesh, M. Richardson, and M. Bass, Opt. Lett. 32, 2505 (2007).

9. W. W. Rigrod, J. Appl. Phys. 34, 2602 (1963).

10. L. W. Casperson and A. Yariv, Appl. Phys. Lett. 12, 355 (1968).

11. F. Caccavale, F. Segato, and I. Mansour, J. Lightwave Technol. 15, 2294 (1997).

12. J. P. Berenger, J. Comput. Phys. 114, 185 (1994).

13. L. W. Casperson, Appl. Opt. 19, 422 (1980). 